\title{
Klein operator and the Number of independent Traces and Supertraces on the Superalgebra of Observables of Rational Calogero Model based on the Root System
}

S.E. Konstein, R. Stekolshchik

To cite this article: S.E. Konstein, R. Stekolshchik (2013) Klein operator and the Number of independent Traces and Supertraces on the Superalgebra of Observables of Rational Calogero Model based on the Root System, Journal of Nonlinear Mathematical Physics 20:2, 295-308, DOI: https://doi.org/10.1080/14029251.2013.820410

To link to this article: https://doi.org/10.1080/14029251.2013.820410

Published online: 04 January 2021 


\title{
Klein operator and the Number of independent Traces and Supertraces on the Superalgebra of Observables of Rational Calogero Model based on the Root System
}

\author{
S.E. Konstein \\ I.E.Tamm Department of Theoretical Physics, P. N. Lebedev Physical Institute, \\ 53, Leninsky Prospect, Moscow, 117924, Russia \\ konstein@lpi.ru \\ R. Stekolshchik \\ r.stekol@gmail.com \\ Received 2 December 2012 \\ Accepted 1 June 2013
}

\begin{abstract}
In the Coxeter group $W(\mathscr{R})$ generated by the root system $\mathscr{R}$, let $T(\mathscr{R})$ be the number of conjugacy classes having no eigenvalue +1 and let $S(\mathscr{R})$ be the number of conjugacy classes having no eigenvalue -1 . The algebra $H_{W(\mathscr{R})}$ of observables of the rational Calogero model based on the root system $\mathscr{R}$ possesses $T(\mathscr{R})$ independent traces; the same algebra, considered as an associative superalgebra with respect to a certain natural parity, possesses $S(\mathscr{R})$ even independent supertraces and no odd trace or supertrace. The numbers $T(\mathscr{R})$ and $S(\mathscr{R})$ are determined for all irreducible root systems (hence for all root systems). It is shown that $T(\mathscr{R}) \leq S(\mathscr{R})$, and $T(\mathscr{R})=S(\mathscr{R})$ if and only if superalgebra $H_{W(\mathscr{R})}$ contains a Klein operator (or, equivalently, $W(\mathscr{R}) \ni-1$ ).
\end{abstract}

Keywords: Trace; supertrace; Cherednik algebra; algebra of observables; Calogero model.

2000 Mathematics Subject Classification: 17B80, 16W55

\section{Definitions and generalities}

\subsection{Traces}

Let $\mathscr{A}$ be an associative superalgebra with parity $\pi$. All expressions of linear algebra are given for homogeneous elements only and are supposed to be extended to inhomogeneous elements via linearity.

A linear function str on $\mathscr{A}$ is called a supertrace if

$$
\operatorname{str}(f g)=(-1)^{\pi(f) \pi(g)} \operatorname{str}(g f) \text { for all } f, g \in \mathscr{A} .
$$

A linear function $\operatorname{tr}$ on $\mathscr{A}$ is called a trace if

$$
\operatorname{tr}(f g)=\operatorname{tr}(g f) \text { for all } f, g \in \mathscr{A} \text {. }
$$

A linear function $L$ is even if $L(f)=0$ for any $f \in \mathscr{A}$ such that $\pi(f)=1$, it is $o d d$ if $L(f)=0$ for any $f \in \mathscr{A}$ such that $\pi(f)=0$. 
Let $\mathscr{A}_{1}$ and $\mathscr{A}_{2}$ be associative superalgebras with parities $\pi_{1}$ and $\pi_{2}$, respectively. Define the tensor product $\mathscr{A}=\mathscr{A}_{1} \otimes \mathscr{A}_{2}$ as a superalgebra with the product $\left(a_{1} \otimes a_{2}\right)\left(b_{1} \otimes b_{2}\right)=\left(a_{1} b_{1}\right) \otimes\left(a_{2} b_{2}\right)$ (no sign factors in this formula) and the parity $\pi$ defined by the formula $\pi(a \otimes b)=\pi_{1}(a)+\pi_{2}(b)$.

Let $T_{i}$ be a trace on $\mathscr{A}_{i}$. Clearly, the function $T$ such that $T(a \otimes b)=T_{1}(a) T_{2}(b)$ is a trace on $\mathscr{A}$.

Let $S_{i}$ be an even supertrace on $\mathscr{A}_{i}$. Clearly, the function $S$ such that $S(a \otimes b)=S_{1}(a) S_{2}(b)$ is an even supertrace on $\mathscr{A}$.

\subsection{Klein operator}

Let $\mathscr{A}$ be an associative superalgebra with parity $\pi$. Following M.Vasiliev, see, e.g. [21], we say that an element $K \in \mathscr{A}$ is a Klein operator ${ }^{\mathrm{a}}$ if $\pi(K)=0, K f=(-1)^{\pi(f)} f K$ for any $f \in \mathscr{A}$ and $K^{2}=1$. Every Klein operator belongs to the anticenter of the superalgebra $\mathscr{A}$, see [18], p.41. ${ }^{\mathrm{b}}$

Any Klein operator, if exists, establishes an isomorphism between the space of even traces and the space of even supertraces on $\mathscr{A}$. Namely, if $f \mapsto T(f)$ is an even trace, then $f \mapsto T(f K)$ is a supertrace, and if $f \mapsto S(f)$ is an even supertrace, then $f \mapsto S(f K)$ is a trace.

\subsection{Group algebra}

Let $V=\mathbb{R}^{n}$ and $G \subset \operatorname{End}(V)$ be a finite group. The group algebra $\mathbb{C}[G]$ of $G$ consists of all linear combinations $\sum_{g \in W(\mathscr{R})} \alpha_{g} \bar{g}$, where $\alpha_{g} \in \mathbb{C}$. We distinguish $g$ considered as an element of the group $G \subset \operatorname{End}(V)$ from the same element $\bar{g} \in \mathbb{C}[G]$ considered as an element of the group algebra. The addition in $\mathbb{C}[G]$ is defined as follows:

$$
\sum_{g \in G} \alpha_{g} \bar{g}+\sum_{g \in G} \beta_{g} \bar{g}=\sum_{g \in G}\left(\alpha_{g}+\beta_{g}\right) \bar{g}
$$

and the multiplication is defined by setting $\overline{g_{1}} \overline{g_{2}}=\overline{g_{1} g_{2}}$.

Note that the additions in $\mathbb{C}[G]$ and in $\operatorname{End}(V)$ differ. For example, if $I \in G$ is unity and the matrix $J=-I$ from $\operatorname{End}(V)$ belongs to $G$, then $I+J=0$ in $\operatorname{End}(V)$ while $\bar{I}+\bar{J} \neq 0$ in $\mathbb{C}[G]$.

\subsection{Root systems}

Let $V=\mathbb{R}^{N}$ be endowed with a non-degenerate symmetric bilinear form $(\cdot, \cdot)$ and the vectors $\vec{a}_{i}$ constitute an orthonormal basis in $V$, i.e.

$$
\left(\vec{a}_{i}, \vec{a}_{j}\right)=\delta_{i j}
$$

Let $x^{i}$ be the coordinates of $\vec{x} \in V$, i.e. $\vec{x}=\vec{a}_{i} x^{i}$. Then $(\vec{x}, \vec{y})=\sum_{i=1}^{N} x^{i} y^{i}$ for any $\vec{x}, \vec{y} \in V$. The indices $i$ are raised and lowered by means of the forms $\delta_{i j}$ and $\delta^{i j}$.

\footnotetext{
$\bar{a}$ In honor of Oskar Klein.

${ }^{\mathrm{b}}$ Let $\mathscr{A}$ be an associative superalgebra with parity $\pi$. Its anticenter $A C(\mathscr{A})$ is defined by the formula

$$
A C(\mathscr{A})=\left\{a \in \mathscr{A} \mid a x-(-1)^{\pi(x)(\pi(a)+1)} x a=0 \text { for any } x \in \mathscr{A}\right\} .
$$
}


For any nonzero $\vec{v} \in V=\mathbb{R}^{N}$, define the reflections $R_{\vec{v}}$ as follows:

$$
R_{\vec{v}}(\vec{x})=\vec{x}-2 \frac{(\vec{x}, \vec{v})}{(\vec{v}, \vec{v})} \vec{v} \quad \text { for any } \vec{x} \in V
$$

The reflections (1.1) have the following properties

$$
R_{\vec{v}}(\vec{v})=-\vec{v}, \quad R_{\vec{v}}^{2}=1, \quad\left(R_{\vec{v}}(\vec{x}), \vec{u}\right)=\left(\vec{x}, R_{\vec{v}}(\vec{u})\right) \quad \text { for any } \vec{v}, \vec{x}, \vec{u} \in V .
$$

A finite set of vectors $\mathscr{R} \subset V$ is said to be a root system if the following conditions hold:

i) $\mathscr{R}$ is $R_{\vec{v}}$-invariant for any $\vec{v} \in \mathscr{R}$,

ii) if $\vec{v}_{1}, \vec{v}_{2} \in \mathscr{R}$ are collinear, then either $\vec{v}_{1}=\vec{v}_{2}$ or $\vec{v}_{1}=-\vec{v}_{2}$. finite.

Clearly, the group $W(\mathscr{R}) \subset O(N, \mathbb{R}) \subset \operatorname{End}(V)$ generated by all reflections $R_{\vec{v}}$ with $\vec{v} \in \mathscr{R}$ is

Let $V=V_{1} \oplus V_{2}$, where $V_{1} \neq\{0\}$ and $V_{2} \neq\{0\}$ are orthogonal with respect to the form $(\cdot, \cdot)$, and let a root system on $V$ have a decomposition: $\mathscr{R}=\mathscr{R}_{1} \bigcup \mathscr{R}_{2}$, where $\mathscr{R}_{i} \subset V_{i}$ for $i=1,2$. Then each $\mathscr{R}_{i} \subset V_{i}$ is a root system. We say in this case that $\mathscr{R}$ is reducible, and denote this fact as $\mathscr{R}=\mathscr{R}_{1}+\mathscr{R}_{2}$. Note, that each $\mathscr{R}_{i}$ can be empty. A root system which is not reducible is called irreducible.

If $\mathscr{R}=\mathscr{R}_{1}+\mathscr{R}_{2}$, then $W(\mathscr{R})=W\left(\mathscr{R}_{1}\right) \times W\left(\mathscr{R}_{2}\right)$.

Any root system has a decomposition $\mathscr{R}=\mathscr{R}_{1}+\mathscr{R}_{2}+\ldots+\mathscr{R}_{n}$, where the $\mathscr{R}_{j}$ are irreducible root systems.

All irreducible root systems are listed in numerous literature (see, e.g., [1], [8], [17], [2], [20], [6], [19]). As it follows from the definition of a root system given above, we consider both crystallographic $\left(A_{n}, B_{n}, C_{n}, D_{n}, E_{6}, E_{7}, E_{8}, F_{4}, G_{2}\right)$ and non-crystallographic $\left(H_{3}, H_{4}, I_{2}(n)\right)$ root systems.

We consider also the empty root system, assuming that it generates the trivial group consisting of the unity element only.

The definition of reducible root system implies that the empty root system in $\mathbb{R}^{N}$ is reducible for any $N>1$. The irreducible empty root system — we denote it $A_{0}$ - belongs to $\mathbb{R}$.

\subsection{The superalgebra of observables}

Let $\mathscr{R}$ be a finite root system. Let $\eta$ be a set of constants $\eta_{\vec{v}}$ with $\vec{v} \in \mathscr{R}$ such that $\eta_{\vec{v}}=\eta_{\vec{w}}$ if $R_{\vec{v}}$ and $R_{\vec{w}}$ belong to one conjugacy class of $W(\mathscr{R})$.

Let $\mathscr{H}^{\alpha}$, where $\alpha=0,1$, be two copies of $V$ with orthonormal bases $a_{\alpha i} \in \mathscr{H}^{\alpha}$, where $i=$ $1, \ldots, N$.

Definition 1.1. The superalgebra $H_{W(\mathscr{R})}(\eta)$ is an associative superalgebra with unity $\mathbf{1}$; it is the superalgebra of polynomials in the $a_{\alpha i}$ with coefficients in the group algebra $\mathbb{C}[W(\mathscr{R})]$ subject to the relations

$$
\begin{aligned}
\bar{g} h_{\alpha} & =g\left(h_{\alpha}\right) h_{\alpha} \bar{g} \text { for any } g \in W(\mathscr{R}) \text { and } h_{\alpha} \in \mathscr{H}^{\alpha}, \\
{\left[x_{\alpha} \bar{I}, y_{\beta} \bar{I}\right] } & =\varepsilon_{\alpha \beta}\left((\vec{x}, \vec{y}) \overline{1} \bar{I}+\sum_{\vec{v} \in \mathscr{R}} \eta_{\vec{v}} \frac{(\vec{x}, \vec{v})(\vec{y}, \vec{v})}{(\vec{v}, \vec{v})} \overline{1} \overline{R_{\vec{v}}}\right) \text { for any } x_{\alpha} \in \mathscr{H}^{\alpha} \text { and } y_{\beta} \in \mathscr{H}^{\beta},
\end{aligned}
$$


where $\varepsilon_{\alpha \beta}$ is the antisymmetric tensor, $\varepsilon_{01}=1$, and $\overline{1}$ is the unity in $\mathbb{C}\left[a_{\alpha i}\right]$. The element $\mathbf{1}=\overline{1} \cdot \bar{I}$ is the unity of $H_{W(\mathscr{R})}(\eta) .{ }^{\mathrm{c}}$ The action of any operator $g \in \operatorname{End}(V)$ is given by a matrix $\left(g_{i}^{j}\right)$ :

$$
\begin{aligned}
g\left(a_{\alpha i} h^{i}\right) & =a_{\alpha i} g_{i}^{j} h^{j}, \quad g_{1}\left(g_{2}\left(h_{\alpha}\right)\right)=\left(g_{1} g_{2}\right)\left(h_{\alpha}\right) \text { for any } h_{\alpha}=a_{\alpha i} h^{i} \in \mathscr{H}^{\alpha}, \\
g(\overline{1}) & =\overline{1} .
\end{aligned}
$$

The commutation relations (1.3) suggest to define the parity $\pi$ by setting:

$$
\pi\left(a_{\alpha i} \bar{g}\right)=1 \text { for any } \alpha, i \text { and } g \in \mathbb{C}[W(\mathscr{R})] ; \quad \pi(\overline{1} \bar{g})=0 \text { for any } g \in \mathbb{C}[W(\mathscr{R})] .
$$

We say that $H_{W(R)}(\eta)$ is a superalgebra of observables of the Calogero model based on the root system $\mathscr{R}$.

Clearly, $H_{W\left(\mathscr{R}_{1}+\mathscr{R}_{2}\right)}(\eta)=H_{W\left(\mathscr{R}_{1}\right)}(\eta) \otimes H_{W\left(R_{2}\right)}(\eta)$.

These algebras $H_{W(R)}(\eta)$ (with parity forgotten) are particular cases of Symplectic Reflection Algebras [3] and are also known as rational Cherednik algebras (see, e.g., [4]).

It follows from eqs. (1.4) and (1.2) that if $I \in W(\mathscr{R}) \subset \operatorname{End}(V)$ is the unity and $J=-I \in \operatorname{End}(V)$ belongs to $W(\mathscr{R})$, then $K:=\overline{1} \bar{J} \in H_{W(\mathscr{R})}(\eta)$ is a Klein operator in $H_{W(\mathscr{R})}(\eta)$.

\section{Traces and supertraces on $H_{W(R)}(\eta)$}

The following facts were proved in [14]:

Theorem 2.1. Let the Coxeter group $W(\mathscr{R}) \subset E n d\left(\mathbb{R}^{N}\right)$ generated by the finite root system $\mathscr{R} \subset \mathbb{R}^{N}$ have $T(\mathscr{R})$ conjugacy classes without eigenvalue 1 and $S(\mathscr{R})$ conjugacy classes without eigenvalue -1 .

Then the superalgebra $H_{W(\mathscr{R})}(\eta)$ possesses $T(\mathscr{R})$ independent traces and $S(\mathscr{R})$ independent supertraces.

Theorem 2.2. Each trace and each supertrace on the superalgebta $H_{W(\mathscr{R})}(\eta)$ is even.

Theorem 2.1 helps to find the numbers $T(\mathscr{R})$ and $S(\mathscr{R})$ for an arbitrary root system $\mathscr{R}$.

Theorem 2.2 implies, clearly, the following statement

Theorem 2.3. In the terms of Theorem 2.1, the following relations are satisfied:

$$
\begin{aligned}
T\left(\mathscr{R}_{1}+\mathscr{R}_{2}\right) & =T\left(\mathscr{R}_{1}\right) T\left(\mathscr{R}_{2}\right), \\
S\left(\mathscr{R}_{1}+\mathscr{R}_{2}\right) & =S\left(\mathscr{R}_{1}\right) S\left(\mathscr{R}_{2}\right) .
\end{aligned}
$$

Therefore, the problem of finding $T(\mathscr{R})$ and $S(\mathscr{R})$ is reduced to the problem of finding $T(\mathscr{R})$ and $S(\mathscr{R})$ for irreducible root systems $\mathscr{R}$.

Here, the number $T(\mathscr{R})$ of traces and the number $S(\mathscr{R})$ of supertraces for all irreducible root systems are found and compared. The result is presented in Sections 3 and 4.

It follows from the results presented in Section 3, that if $T(\mathscr{R})=S(\mathscr{R})$ for some irreducible root system $\mathscr{R}$, then $-I \in W(\mathscr{R})$, and so $H_{W(\mathscr{R})}(\eta)$ has a Klein operator.

The results of this paper were preprinted in [13] and [12].

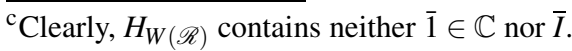


3. The numbers $T(\mathscr{R})$ of traces and $S(\mathscr{R})$ of supertraces for irreducible root system $\mathscr{R}$ if $T(\mathscr{R})=S(\mathscr{R})$

\begin{tabular}{|c|c|c|c|}
\hline $\mathscr{R}$ & $T(\mathscr{R})=S(\mathscr{R})$ & $\begin{array}{l}\text { presence of }-I \\
\text { in } W(\mathscr{R}) \text { proved in }\end{array}$ & proof in: \\
\hline$A_{1}$ & 1 & [1], Table I (XI) & Appendix A.2 \\
\hline$B_{n}, C_{n}$ & $\begin{array}{l}\text { the number of partitions } \\
\text { of } n \text { into the sum of } \\
\text { positive integers }\end{array}$ & [1], Tables II, III (XI) & Appendix A.3 \\
\hline$D_{2 n}$ & $\begin{array}{l}\text { the number of partitions } \\
\text { of } 2 n \text { into the sum of } \\
\text { positive integers with } \\
\text { an even number of summands }\end{array}$ & [1], Table IV (XI) & Appendix A.4 \\
\hline$E_{7}$ & 12 & [1], Table VI (XI) & Appendix A.6 \\
\hline$E_{8}$ & 30 & [1], Table VII (XI) & Appendix A.7 \\
\hline$F_{4}$ & 9 & [1], Table VIII (XI) & Appendix A.8 \\
\hline$G_{2}$ & 3 & [1], Table IX (XI) & Appendix A.9 \\
\hline $\mathrm{H}_{3}$ & 4 & [6], p.160; Appendix A.10 & Appendix A.10 \\
\hline$H_{4}$ & 20 & [6], Table $3, K_{2}$ & Appendix A.11 \\
\hline$I_{2}(2 n)$ & $n$ & Appendix A.12 & Appendix A.12 \\
\hline
\end{tabular}

4. The numbers $T(\mathscr{R})$ of traces and $S(\mathscr{R})$ of supertraces for irreducible root system $\mathscr{R}$ if $W(R) \not \supset-I$

\begin{tabular}{|c|c|c|c|}
\hline $\mathscr{R}$ & $T(\mathscr{R})$ & $S(\mathscr{R})$ & proof in: \\
\hline \hline$A_{0}$ & 0 & 1 & Appendix A.1 \\
\hline$A_{n-1}, n \geq 3$ & 1 & $\begin{array}{l}\text { the number of partitions } \\
\text { of } n \text { into the sum of odd } \\
\text { positive integers }\end{array}$ & Appendix A.2 \\
\hline$D_{2 n+1}$ & $\begin{array}{l}\text { the number of partitions } \\
\text { of } 2 n+1 \text { into the sum of } \\
\text { positive integers with } \\
\text { an even number of summands }\end{array}$ & $\begin{array}{l}\text { the number of partitions } \\
\text { of } 2 n+1 \text { into the sum of } \\
\text { positive integers with } \\
\text { an odd number of summands }\end{array}$ & Appendix A.4 \\
\hline$E_{6}$ & 5 & 9 & Appendix A.5 \\
\hline$I_{2}(2 n+1)$ & $n$ & $n+1$ & Appendix A.12 \\
\hline
\end{tabular}

The Weyl superalgebra. Let $W_{n}$ be Weyl superalgebra with $n$ pairs of generating elements: $W_{n}=\mathbb{C}\left[a_{\alpha i}\right]$, where $\alpha=0,1$ and $i=1, \ldots, n$, subject to relations $\left[a_{\alpha i}, a_{\beta j}\right]=\varepsilon_{\alpha \beta} \delta_{i j}$ and with parity defined by $\pi\left(a_{\alpha i}\right)=1$. Clearly, $W_{n}=\left(W_{1}\right)^{\otimes n}$. Further, superalgebra $H_{W\left(A_{0}\right)}(\eta)$ does not depend on $\eta$ and since $A_{0}$ is irreducible, $H_{W\left(A_{0}\right)}(\eta)=W_{1}$. So, due to first row of Table 4 and Theorem 2.3, the Weyl superalgebra $W_{n}$ has 1 supertrace and 0 traces.

\section{Inequality Theorem}

Theorem 5.1. Let $H_{W(\mathscr{R})}(\eta)$ has $T(\mathscr{R})$ traces and $S(\mathscr{R})$ supertraces. Then 
i) $S(\mathscr{R})>0$,

ii) $T(\mathscr{R}) \leq S(\mathscr{R})$,

iii) $T(\mathscr{R})=S(\mathscr{R})$ if and only if $W(\mathscr{R})$ contains $-I$. Equivalently, $T(\mathscr{R})=S(\mathscr{R})$ if and only if $H_{W(\mathscr{R})}(\eta)$ contains a Klein operator.

Proof. Since each group contains unity $I$ and spectrum of $I$ does not contain -1 , it follows that $S(\mathscr{R})>0$.

Let $K \in H_{W(\mathscr{R}}(\eta)$ be a Klein operator. Then $K$ establishes one-to-one correspondence between traces and supertraces:

$$
\operatorname{tr}(f)=\operatorname{str}(f K), \mathrm{s} \operatorname{tr}(g)=\operatorname{tr}(g K)
$$

Let $T(\mathscr{R})=S(\mathscr{R})$. Then the decomposition of $\mathscr{R}$ in the sum of irreducible root systems does not contain root systems from Table 4, namely $A_{0}, A_{n}$ for $n \geq 2, D_{2 n+1}$ for $n \geq 1$ and $E_{6}$, because $T\left(\mathscr{R}_{i}\right)<S\left(\mathscr{R}_{i}\right)$ for all these root systems. So, this decomposition contains the root systems listed in Table 3 only, each of these groups has the element $-I$ and the direct product of all these $-I \mathrm{~s}$ is $-I$ in $W(\mathscr{R})$.

It remains to prove the inequalities

$$
\begin{aligned}
& T\left(A_{n}\right)<S\left(A_{n}\right) \quad \text { for } n=0 \text { and } n \geq 2, \\
& T\left(D_{2 n+1}\right)<S\left(D_{2 n+1}\right) \text { for } n \geq 1, \\
& T\left(E_{6}\right)<S\left(E_{6}\right) .
\end{aligned}
$$

Inequalities (5.1) and (5.3) manifestly follow from Table 4, and inequality (5.2) follows from Table 4 and Lemma 5.1, ii) below.

Lemma 5.1. ${ }^{\mathrm{d}}$ Let $E(n)$ be the number of partitions of $n$ into the sum of positive integers with an even number of summands. Let $O(n)$ be the number of partitions of $n$ into the sum of positive integers with an odd number of summands. Then

i) $E(2 k)>O(2 k)$ for $k \geq 2$,

ii) $E(2 k-1)<O(2 k-1)$ for $k \geq 1$,

iii) $E(2)=O(2)$,

iv) $|E(n)-O(n)|=R(n)$, where $R(n)$ is the number of partitions of $n$ into the sum of different positive odd integers.

Proof. Let $a_{m n}$ be the number of partitions of $n$ into the sum of positive integers with $m$ summands, $a_{m 0}=\delta_{m 0}$. Introduce the generating function

$$
F(t, x)=\sum_{m, n=0}^{\infty} a_{m n} t^{m} x^{n}
$$

Then

$$
\sum_{n} E(n) x^{n}=\left.\frac{1}{2}(F(t, x)+F(-t, x))\right|_{t=1} \text { and } \sum_{n} O(n) x^{n}=\left.\frac{1}{2}(F(t, x)-F(-t, x))\right|_{t=1} .
$$

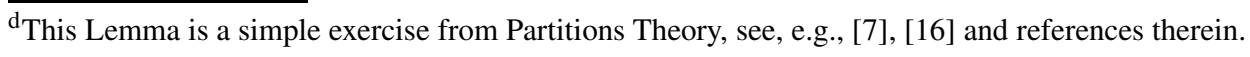


Hence,

$$
\sum_{n}(O(n)-E(n)) x^{n}=-\left.F(-t, x)\right|_{t=1}
$$

Further,

$$
\begin{aligned}
F(t, x) & =\left(1+t x+(t x)^{2}+(t x)^{3}+\cdots\right)\left(1+t x^{2}+\left(t x^{2}\right)^{2}+\left(t x^{2}\right)^{3}+\ldots\right) \ldots \\
& =\frac{1}{(1-t x)\left(1-t x^{2}\right)\left(1-t x^{3}\right) \ldots}
\end{aligned}
$$

So

$$
-\left.F(-t, x)\right|_{t=1}=-\frac{1}{(1+x)\left(1+x^{2}\right)\left(1+x^{3}\right) \ldots}
$$

Multiplying both terms of fraction (5.4) by $(1-x)\left(1-x^{2}\right)\left(1-x^{3}\right) \ldots$ we obtain

$$
-\left.F(-t, x)\right|_{t=1}=-\frac{(1-x)\left(1-x^{2}\right)\left(1-x^{3}\right) \ldots}{\left(1-x^{2}\right)\left(1-x^{4}\right)\left(1-x^{6}\right) \ldots}=-(1-x)\left(1-x^{3}\right)\left(1-x^{5}\right) \ldots
$$

Now, it suffices to notice that eq. (5.5) can be rewritten in the form

$$
\sum_{n}(O(n)-E(n)) x^{n}=-\left.F(-t, x)\right|_{t=1}=\sum_{n \text { is odd }} R(n) x^{n}-\sum_{n \text { is even }} R(n) x^{n}
$$

\section{Acknowledgments}

This work was supported by the Russian Foundation for Basic Research, Grant No. 11-02-00685.

\section{Appendix A. Computing the number of traces and supertraces for all irreducible root systems}

\section{A.1. Root system $A_{0}$}

The Weyl algebra $\mathbb{C}\left[a, a^{+}\right]$generated by elements $a$ and $a^{+}$satisfying the relation $\left[a, a^{+}\right]=1$ may be considered as the algebra of observables of the Calogero model based on the empty irreducible root system $A_{0}$, which generates the trivial group consisting only of the unity 1 . This group has 1 conjugacy class without -1 in its spectrum and 0 conjugacy classes without 1 in its spectrum. So, this Weyl algebra has 0 traces and 1 supertrace.

\section{A.2. Root systems $A_{n-1}$ for $n>1$}

It is well-known that $W\left(A_{n-1}\right)=S_{n}$ and $V=\operatorname{span}\left(e_{1}-e_{2}, e_{2}-e_{3}, \ldots, e_{n-1}-e_{n}\right)$. Each element of $S_{n}$ can be decomposed in the product of cycles of the form

$$
\sigma: e_{i_{1}} \rightarrow e_{i_{2}} \rightarrow \ldots \rightarrow e_{i_{k}} \rightarrow e_{i_{1}} .
$$




\section{A.2.1. The number of traces}

If $k<n$, then each cycle $\sigma: e_{i_{1}} \rightarrow e_{i_{2}} \rightarrow \ldots \rightarrow e_{i_{k}} \rightarrow e_{i_{1}}$ has eigenvalue +1 with eigenvector

$$
e_{i_{1}}+e_{i_{2}}+\ldots+e_{i_{k}}-\frac{k}{n} \sum_{s=1}^{n} e_{s} .
$$

The only conjugacy class without eigenvalue +1 is the one containing the cycle of maximal length $n$ :

$$
\sigma: e_{1} \rightarrow e_{2} \rightarrow \ldots \rightarrow e_{n} \rightarrow e_{1}
$$

because its characteristic polynomial has the form

$$
f(t)=1+t+\ldots+t^{n-1} .
$$

\section{A.2.2. The number of supertraces (see also [15])}

The cycle $\sigma: e_{i_{1}} \rightarrow e_{i_{2}} \rightarrow \ldots \rightarrow e_{i_{k}} \rightarrow e_{i_{1}}$ has eigenvalue -1 if and only if $k$ is even. The corresponding eigenvector has the form

$$
e_{i_{1}}-e_{i_{2}}+\ldots-e_{i_{k}} \text {. }
$$

So, the number of conjugacy classes without eigenvalue -1 is equal to the number of partitions of $n$ into the sum of positive odd integers [15].

A.2.3. Presence of $-I$ in the group $W\left(A_{n-1}\right)$, where $n>1$.

If $A_{n-1} \ni-I$, then $n=2$ (see [1], Table I (XI)). The group $W\left(A_{1}\right)$ consists of two elements: $I$ and $-I$.

\section{A.3. Root systems $B_{n}$ and $C_{n}$}

The Coxeter group $G=W\left(B_{n}\right)=W\left(C_{n}\right)$ is generated by the permutation group $S_{n}$ and reflections $R_{i}: e_{i} \rightarrow-e_{i}, e_{j} \rightarrow e_{j}$ for $i \neq j$, see [1]. Each element $g \in G$ can be represented in the form

$$
g=\sigma \prod_{i=1}^{n} R_{i}^{\alpha_{i}}, \text { where } \sigma \in S_{n} \text { and } \alpha_{i} \in\{0,1\} .
$$

The set $\left(\sigma, \alpha_{1}, \ldots \alpha_{n}\right)$ unambiguously defines every element of $G$.

Since each permutation can be decomposed in the product of commuting cycles,

$$
\sigma=\prod \hat{\sigma}_{k}, \quad \text { where } \hat{\sigma}_{k}: e_{i_{1}} \rightarrow e_{i_{2}} \rightarrow \ldots \rightarrow e_{i_{k}} \rightarrow e_{i_{1}},
$$

we can introduce what we call $R$-cycles by the formula

$$
\tilde{\sigma}_{k}=\hat{\sigma}_{k} R_{i_{1}}^{\alpha_{i_{1}}} R_{i_{2}}^{\alpha_{i_{2}}} \ldots R_{i_{k}}^{\alpha_{i_{k}}}
$$

So, each element $g \in G$ has the form

$$
g=\prod_{p} \tilde{\sigma}_{p}
$$

We say that the value $\varepsilon_{R}(\tilde{\sigma})=\left|\alpha_{i_{1}}+\alpha_{i_{2}} \ldots+\alpha_{i_{k}}\right|_{\bmod 2}$ is the $R$-parity of the $R$-cycle $\tilde{\sigma}$. Let $l(\tilde{\sigma})=k$, where $k$ is the length of the cycle $\hat{\sigma}$. 
It is easy to prove that an $R$-cycle $\tilde{\sigma}_{1}$ is conjugated to an $R$-cycle $\tilde{\sigma}_{2}$ if and only if $l\left(\tilde{\sigma}_{1}\right)=l\left(\tilde{\sigma}_{2}\right)$ and $\varepsilon_{R}\left(\tilde{\sigma}_{1}\right)=\varepsilon_{R}\left(\tilde{\sigma}_{2}\right)$.

So, a conjugacy class in $G$ is characterized by the numbers $p_{1}, p_{2}, \ldots, p_{n}$ and $q_{1}, q_{2}, \ldots, q_{n}$, where $p_{i}$ is the number of $R$-cycles of length $i$ and $R$-parity 0 , and $q_{i}$ is the number of $R$-cycles of length $i$ and $R$-parity 1 , in the presentation of $g$ in the form (A.1).

The numbers $p_{i}$ and $q_{i}$ satisfy the relation

$$
\sum_{i=1}^{n}\left(i p_{i}+i q_{i}\right)=n
$$

\section{A.3.1. The number of traces}

The characteristic polynomial of the $R$-cycle $\tilde{\sigma}$ has the form

$$
(-1)^{l(\tilde{\sigma})}\left(t^{l(\tilde{\sigma})}-(-1)^{\varepsilon(\tilde{\sigma})}\right) .
$$

It has no root +1 if $\varepsilon(\tilde{\sigma})=1$.

So, if given conjugacy class has no eigenvalue +1 , then $p_{i}=0$ and $\sum_{i} i q_{i}=n$.

\section{A.3.2. The number of supertraces (see also [9])}

The characteristic polynomial of an $R$-cycle $\tilde{\sigma}$ (A.3) has no root -1 if either $l(\tilde{\sigma})$ is even and $\varepsilon(\tilde{\sigma})=1$ or if $l(\tilde{\sigma})$ is odd and $\varepsilon(\tilde{\sigma})=0$.

So, if a given conjugacy class has no eigenvalue -1 , then $p_{2 k}=0$ and $q_{2 k+1}=0$ and eq. (A.2) gives $p_{1}+2 q_{2}+3 p_{3}+4 q_{4}+\ldots=n$.

\section{A.3.3. Presence of $-I$ in the group $W\left(B_{n}\right)$.}

It is easy to see that $-I=\prod_{i=1}^{n} R_{i}$.

\section{A.4. Root systems $D_{n}$}

The Coxeter group $W\left(D_{n}\right)$ is a subgroup of $W\left(B_{n}\right)$, namely, $g=\prod \tilde{\sigma}_{s}$ belongs to $W\left(D_{n}\right)$ if $\left.\left(\sum_{s} \varepsilon_{R}\left(\tilde{\sigma}_{s}\right)\right)\right|_{\bmod 2}=0[1]$.

\section{A.4.1. The number of traces}

So, $g$ has no eigenvalue +1 if $p_{i}=0, \sum_{i} i m_{i}=n$ and $\left.\left(\sum_{i} m_{i}\right)\right|_{\bmod 2}=0$.

This implies that $T\left(D_{n}\right)$ is equal to the number of partition of $n$ into the sum of positive integers with an even number of summands.

\section{A.4.2. The number of supertraces (see also [9])}

Analogously, $S\left(D_{n}\right)$ is equal to the number of partitions of $n$ into the sum of positive integers with an even number of even integers.

Clearly, if $n$ is even, then $S\left(D_{n}\right)$ is equal to the number of partitions of $n$ into the sum of positive integers with an even number of summands, and if $n$ is odd, then $S\left(D_{n}\right)$ is equal to the number of partitions of $n$ into the sum of positive integers with an odd number of summands. 
A.4.3. Presence of $-I$ in the group $W\left(D_{n}\right)$.

If $n$ is even, then $-I=\prod_{k=1}^{n} R_{k}$.

\section{A.5. Root system $E_{6}$}

The conjugacy classes of the Weyl group $E_{6}$ are described in Table 9 of [2] .

\section{A.5.1. The number of traces}

The following 5 classes do not have the root +1 :

$$
A_{2}^{3}, A_{5} \times A_{1}, E_{6}, E_{6}\left(a_{1}\right), E_{6}\left(a_{2}\right) .
$$

\section{A.5.2. The number of supertraces}

The following 9 classes do not have the root -1 :

$$
\phi, A_{2}, A_{2}^{2}, A_{4}, D_{4}\left(a_{1}\right), A_{2}^{3}, E_{6}, E_{6}\left(a_{1}\right), E_{6}\left(a_{2}\right) .
$$

\section{A.6. Root system $E_{7}$}

The conjugacy classes of the Weyl group $E_{7}$ are described in Table 10 of [2] .

\section{A.6.1. The number of traces}

The last 12 classes in Table 10 of [2] do not have the root +1 :

$$
\begin{aligned}
& A_{1}^{7}, A_{3}^{2} \times A_{1}, A_{5} \times A_{2}, A_{7}, D_{4} \times A_{1}^{3}, D_{6} \times A_{1}, D_{6}\left(a_{2}\right) \times A_{1}, \\
& E_{7}, E_{7}\left(a_{1}\right), E_{7}\left(a_{2}\right), E_{7}\left(a_{3}\right), E_{7}\left(a_{4}\right) .
\end{aligned}
$$

\section{A.6.2. The number of supertraces}

The following 12 classes do not have the root -1 :

$$
\phi, A_{2}, A_{2}^{2}, A_{4}, D_{4}\left(a_{1}\right), A_{2}^{3}, A_{4} \times A_{2}, A_{6}, D_{6}\left(a_{1}\right), E_{6}, E_{6}\left(a_{1}\right), E_{6}\left(a_{2}\right) .
$$

\section{A.7. Root system $E_{8}$}

The conjugacy classes of the Weyl group $E_{8}$ are described in Table 11 of [2] .

\section{A.7.1. The number of traces}

The last 30 classes in Table 11 of [2] do not have the root +1 :

$$
\begin{aligned}
& A_{1}^{8}, A_{2}^{4}, A_{3}^{2} \times A_{1}^{2}, A_{4}^{2}, A_{5} \times A_{2} \times A_{1}, A_{7} \times A_{1}, A_{8}, D_{4} \times A_{1}^{4}, D_{4}^{2}, D_{4}\left(a_{1}\right)^{2}, \\
& D_{5}\left(a_{1}\right) \times A_{3}, D_{6} \times A_{1}^{2}, D_{8}, D_{8}\left(a_{1}\right), D_{8}\left(a_{2}\right), D_{8}\left(a_{3}\right), E_{6} \times A_{2}, E_{6}\left(a_{2}\right) \times A_{2}, \\
& E_{7} \times A_{1}, E_{7}\left(a_{2}\right) \times A_{1}, E_{7}\left(a_{4}\right) \times A_{1}, E_{8}, E_{8}\left(a_{i}\right)(i=1, \ldots 8) .
\end{aligned}
$$

\footnotetext{
${ }^{\mathrm{e}}$ In [2], the Carter diagrams are used to describe the conjugacy classes of the finite Weyl groups. Additional results on the Carter diagrams are recently obtained in [20]. In particular, two problems are discussed:

i) two Carter diagrams can correspond to one conjugacy class ([20], Theorem 4.1) and

ii) certain Carter diagrams can correspond to two distinct conjugacy classes ([20], Theorem 6.5).
} 


\section{A.7.2. The number of supertraces}

The following 30 classes do not have the root -1 :

$$
\begin{aligned}
& \phi, A_{2}, A_{2}^{2}, A_{4}, D_{4}\left(a_{1}\right), A_{2}^{3}, A_{4} \times A_{2}, A_{6}, D_{4}\left(a_{1}\right) \times A_{2}, D_{6}\left(a_{1}\right), \\
& E_{6}, E_{6}\left(a_{1}\right), E_{6}\left(a_{2}\right), A_{2}^{4}, A_{4}^{2}, A_{8}, D_{4}\left(a_{1}\right)^{2}, D_{8}\left(a_{1}\right), D_{8}\left(a_{3}\right), \\
& E_{6} \times A_{2}, E_{6}\left(a_{2}\right) \times A_{2}, E_{8}, E_{8}\left(a_{i}\right)(i=1, \ldots 8) .
\end{aligned}
$$

\section{A.8. Root system $F_{4}$}

The conjugacy classes of the Weyl group $F_{4}$ are described in Table 8 of [2] .

\section{A.8.1. The number of traces}

The last 9 classes in Table 8 of [2] do not have the root +1 :

$$
A_{1}^{4}, A_{2} \times \tilde{A}_{2}, A_{3} \times \tilde{A}_{1}, C_{3} \times A_{1}, D_{4}, D_{4}\left(a_{1}\right), B_{4}, F_{4}, F_{4}\left(a_{1}\right) .
$$

\section{A.8.2. The number of supertraces}

The following 9 classes do not have the root -1 :

$$
\phi, A_{2}, \tilde{A}_{2}, B_{2}, A_{2} \times \tilde{A}_{2}, D_{4}\left(a_{1}\right), B_{4}, F_{4}, F_{4}\left(a_{1}\right) .
$$

\section{A.9. Root system $G_{2}$}

The conjugacy classes of the Weyl group $G_{2}$ are described in Table 7 of [2] $]^{e}$.

\section{A.9.1. The number of traces}

The last 3 classes in Table 7 of [2] do not have the root +1 :

$$
A_{1} \times \tilde{A}_{1}, A_{2}, G_{2},
$$

\section{A.9.2. The number of supertraces (see [10])}

The following 3 classes in Table 7 of [2] do not have the root -1 :

$$
\phi, A_{2}, G_{2},
$$

The fact that $G_{2}$ has 3 conjugacy classes without eigenvalue +1 and 3 conjugacy classes without eigenvalue -1 can be derived also from Appendix A.12 because $G_{2}=I_{2}(6)$. 


\section{A.10. Root system $\mathrm{H}_{3}$}

Let $k=\frac{1}{2}(\sqrt{5}+1)$. Then the reflections

$$
a=\left(\begin{array}{ccc}
1 & 0 & 0 \\
0 & -1 & 0 \\
0 & 0 & 1
\end{array}\right), \quad b=\frac{1}{2}\left(\begin{array}{ccc}
1 & k & k-1 \\
k & 1-k & -1 \\
k-1 & -1 & k
\end{array}\right), \quad c=\left(\begin{array}{ccc}
-1 & 0 & 0 \\
0 & 1 & 0 \\
0 & 0 & 1
\end{array}\right)
$$

corresponding to the roots $\vec{e}_{2}, \frac{1}{2}\left(-\vec{e}_{1}+k \vec{e}_{2}+k^{-1} \vec{e}_{3}\right)$ and $\vec{e}_{1}$, respectively, satisfy the relations

$$
a^{2}=b^{2}=c^{2}=1,(a b)^{5}=(b c)^{3}=(a c)^{2}=1
$$

and generate the Coxeter group $\mathrm{H}_{3}$.

As $H_{3}=S_{5}^{e} \times C_{2}$ (See [1], Ch.VI, Sect. 4, Ex.11 d), p. 284; [6], p.160 and references therein), where $S_{5}^{e}$ is the group of even permutations of 5 elements, $C_{2}=\{1,-1\}$, it follows that $H_{3}$ has 10 conjugacy classes, 5 with a positive determinant and 5 with a negative one. (Observe that $\left|S_{5}^{e}\right|=60$, hence $\left|H_{3}\right|=120$.)

The conjugacy classes with positive determinant are described by their representatives

\begin{tabular}{|l|l|}
\hline The representative & The characteristic polynomial \\
\hline$I$ & $(1-t)^{3}$ \\
$a c$ & $(1-t)(1+t)^{2}$ \\
$b c$ & $(1-t)\left(t^{2}+t+1\right)$ \\
$a b$ & $(1-t)\left[t^{2}+(1-k) t+1\right]$ \\
$a b a b$ & $(1-t)\left(t^{2}+k t+1\right)$ \\
\hline
\end{tabular}

\section{A.10.1. The number of supertraces}

Only four conjugacy classes have no roots -1 , their representatives are $I, b c, a b$ and $a b a b$.

\section{A.10.2. The number of traces}

Each of the characteristic polynomials (A.4) has the root +1 . Besides, the conjugacy class with representative $-a c$ has the root +1 , so only four conjugacy classes with negative determinant have no roots +1 . Their representatives are $-I,-b c,-a b$ and $-a b a b$.

So, the number of conjugacy classes without root +1 is equal to 4 .

A.10.3. Presence of $J=-I$ in $W\left(H_{3}\right)$.

The group $H_{3}$ contains the element $J=-I=(a b a b c)^{3}$ (see [5], p.11). 


\section{A.11. Root system $H_{4}$}

According to [6], all 34 conjugacy classes of the Coxeter group $H_{4}$ are described by their representatives acting on the space of quaternions

$$
\begin{aligned}
& g_{l r}: x \mapsto l x r^{*}, \\
& g_{p}^{*}: x \mapsto p x^{*} .
\end{aligned}
$$

All 25 pairs of unit quaternions $l$ and $r$ and 9 unit quaternions $p$ are listed in Table 3 of [6].

\section{A.11.1. The number of traces}

Each operator (A.6) has the root +1 . Indeed, the equation $p x^{*}=x$ has a nonzero solution $x=1+p$ if $p \neq-1$, and $x$ is an arbitrary imaginary quaternion if $p=-1$.

Each operator (A.5) does not have the root +1 if and only if

$$
l_{0}-r_{0} \neq 0 .
$$

Indeed, the determinant of the map $x \mapsto l x-x r$ is equal to $4\left(l_{0}-r_{0}\right)^{2}$.

There are 20 pairs $(l, r)$ in Table 3 of [6] satisfying the condition (A.7), namely, $K_{i}$ with $i=2,5$, 7,9 to 25 .

\section{A.11.2. The number of supertraces}

Each operator (A.6) has the root -1 . Indeed, the equation $p x^{*}=-x$ has nonzero solution $x=1-p$ if $p \neq 1$, and $x$ is an arbitrary imaginary quaternion if $p=1$.

Each operator (A.5) does not have the root -1 if and only if

$$
l_{0}+r_{0} \neq 0 \text {. }
$$

Indeed, the determinant of the map $x \mapsto l x-x r$ is equal to $4\left(l_{0}+r_{0}\right)^{2}$.

There are 20 pairs of $l, r$ in Table 3 of [6] satisfying the condition (A.8), namely, $K_{i}$ with $i=1$, $4,6,8,10$ to 25 .

\section{A.11.3. Presence of element $J=-I$ in $W\left(H_{4}\right)$.}

The element $K_{2}$ in Table 3 of [6] with $l=-r=1$ is $-I$ in $H_{4}$.

\section{A.12. Root systems $I_{2}(n)$}

It is convenient to use $\mathbb{C}$ instead of $\mathbb{R}^{2}$ to describe $W\left(I_{2}(n)\right)$. The root system $I_{2}(n)$ contains $2 n$ vectors $v_{k}=\exp (\pi i k / n)$, where $k=0,1, \ldots, 2 n-1$. The corresponding Coxeter group $W\left(I_{2}(n)\right)$ has $2 n$ elements, $n$ reflections $R_{k}$ acting on $z, z^{*} \in \mathbb{C}$ as follows

$$
\begin{aligned}
R_{k} z & =-z^{*} v_{k}^{2} R_{k}, \\
R_{k} z^{*} & =-z v_{k}^{* 2} R_{k}, \quad k \in \mathbb{Z}_{n}
\end{aligned}
$$

and $n$ elements of the form $S_{k}=R_{k} R_{0}$, where $S_{0}$ is the unity in $W\left(I_{2}(n)\right)$. These elements satisfy the following relations

$$
R_{k} R_{l}=S_{k-l}, \quad S_{k} S_{l}=S_{k+l}, \quad R_{k} S_{l}=R_{k-l}, \quad S_{k} R_{l}=R_{k+l} .
$$


Obviously, the reflections $R_{2 k}$ lie in one conjugacy class and $R_{2 k+1}$ lie in another one if $n$ is even. If $n$ is odd, then all reflections $R_{k}$ lie in one conjugacy class. Each reflection has both eigenvalues +1 and -1 .

The rotation $S_{k}$ has no eigenvalues -1 if $k \neq n / 2$, and has no eigenvalues +1 if $k \neq 0$. If $n$ is even, then $S_{n / 2}=-I$.

Rotations $S_{k}$ and $S_{-k}$ form a conjugacy class.

So, the number of conjugacy classes without +1 is equal to $\left[\frac{n}{2}\right]$, and the number of conjugacy classes without -1 is equal to $\left[\frac{n+1}{2}\right]$, see [10].

\section{References}

[1] N. Bourbaki, Groupes et algèbres de Lie, ch.IV-VI, Hermann, Paris, 1968.

[2] R.W. Carter, "Conjugacy classes in the Weyl group", Compositio Mathematica, $25 \mathrm{n}^{\circ} 1$, (1972) 1-59.

[3] P. Etingof and V. Ginzburg, "Symplectic reflection algebras, Calogero-Moser space, and deformed Harish-Chandra homomorphism”, Inv. Math. 147(2002), 243-348; arXiv:math.AG/0011114.

[4] P. Etingof, Xiaoguang Ma, "Lecture notes on Cherednik algebras", arXiv:1001.0432v4.

[5] W.N. Franzsen, "Automorphisms of Coxeter Groups", A thesis for the degree of Doctor of Philosophy, School of Mathematics and Statistics University of Sydney, January, 2001.

[6] L.C. Grove, "The characters of the hecatonicosahedroidal group", Journal für die reine und angewandte Mathematik, 265 (1974) 160-169.

[7] M. Hall, Combinatorial theory, 2nd edition, Wisley, New York (1986), 464 pp.

[8] J.E. Humphreys, Reflection groups and Coxeter groups, Cambridge University Press, 1990.

[9] S.E. Konstein, Teor. Mat. Fiz., 111 (1997) 252-262.

[10] S.E. Konstein, "Rational Calogero models based on rank-2 root systems: supertraces on the superalgebras of observables", arXiv:math/9801001.

[11] S.E. Konstein, "Supertraces on the Superalgebra of Observables of Rational Calogero Model based on the Root System", arXiv:math-ph/9904032.

[12] S.E. Konstein, "Klein operator and the Numbers of independent Traces and Supertraces on the Superalgebra of Observables of Rational Calogero Model based on the Root System", arXiv:1212.0508.

[13] S.E. Konstein and R. Stekolshchik, "The Number of Supertraces on the Superalgebra of Observables of Rational Calogero Model based on the Root System", arXiv:0811.2487.

[14] S.E. Konstein and I.V. Tyutin, "Traces on the Superalgebra of Observables of Rational Calogero Model based on the Root System", Journal of Nonlinear Mathematical Physics, Vol. 20, No. 2 (June 2013), 271-294; arXiv:1211.6600.

[15] S.E. Konstein and M.A. Vasiliev, J. Math. Phys. 37 (1996) 2872

[16] S.K. Lando, Lectures on generating functions. Series: Student mathematical library, v. 23, Published 2003 by American Mathematical Society in Providence, RI, 144 pp.

[17] M.A. Olshanetsky and A.M. Perelomov, Phys. Rep., 94 (1983) 313.

[18] Seminar on supersymmetry (v. 1. Algebra and Calculus: Main chapters), (J. Bernstein, D. Leites, V. Molotkov, V. Shander) ed. by D. Leites, MCCME, Moscow, 2011, 410 pp (in Russian; a version in English is in preparation).

[19] R. Stekolshchik, Notes on Coxeter Transformatios and the McKay Correspondence. Series: Springer Monographs in Mathematics 2008, XX, 239 pp.

[20] R. Stekolshchik, "Root systems and diagram calculus. I. Regular extensions of Carter diagrams and the uniqueness of conjugacy classes", arXiv:1005.2769v6.

[21] M.A. Vasiliev, JETP Letters, 50 (1989) 344-347; Int. J. Mod. Phys. A6 (1991) 1115. 